Pure Appl. Chem., Vol. 82, No. 6, pp. 1239-1245, 2010.

doi:10.1351/PAC-CON-09-10-38

(C) 2010 IUPAC, Publication date (Web): 20 April 2010

\title{
Plasma functionalization of textiles: Specifics and possibilities*
}

\author{
Sebastien Guimond ${ }^{\ddagger}$, Barbara Hanselmann, Martin Amberg, and \\ Dirk Hegemann
}

\begin{abstract}
Empa, Swiss Federal Laboratories for Materials Testing and Research, Lerchefenldstrasse 5, St. Gallen, Switzerland
\end{abstract}

\begin{abstract}
Plasma technology offers many interesting possibilities for the production of highvalue-added textiles. Nevertheless, textiles can have a considerable structural and chemical complexity, and their properties must be taken into account for the implementation of plasma processes. The influences of some of these properties are highlighted through several examples of recent interesting applications, such as the metallization of polyester yarns, the enhancement of fabric moisture wicking and the surface functionalization with plasma polymerization.
\end{abstract}

Keywords: metallization; plasma activation; plasma polymerization; textiles; wicking.

\section{INTRODUCTION}

A shift toward highly functional and added-value textiles is now recognized as being essential to the sustainable growth of the textile and clothing industry in developed countries. The demand for tailored surface modifications for water repellence, long-term hydrophilicity, enhanced adhesion, antibacterial properties, etc., is therefore increasing. At the same time, the environmental restrictions concerning waste water produced by conventional textile finishing techniques are becoming more and more severe, generating higher running costs. In this context, plasma processing is seen as a very attractive alternative method to add new functionalities to textiles [1,2]. Plasma processing is a clean (dry) and sustainable technology that generates minimal amounts of waste. It is also characterized by much lower materials and energy consumption in comparison to wet chemical-based finishing methods, potentially resulting in markedly reduced running costs. Furthermore, plasma processing is very versatile since it can be used to impart a broad range of different properties, some of which is unattainable with conventional methods. It can also be applied to both individual yarns and fabrics. Finally, because plasma processing results in a nanoscaled surface modification, it has the advantage of preserving the bulk properties as well as the touch of textiles.

With examples taken from the research done at Empa, this contribution aims at giving an overview of some of the properties specific to textiles and their impact on related plasma processes and applications. Interesting possibilities offered by the application of plasma technology to textiles are also outlined.

\footnotetext{
*Paper based on a presentation at the $19^{\text {th }}$ International Symposium on Plasma Chemistry (ISPC-19), 26-31 July 2009, Bochum, Germany. Other presentations are published in this issue, pp. 1189-1351.

¥Corresponding author
} 


\section{SURFACE CLEANING AND METALLIZATION}

The structure and surface chemical composition of textiles usually vary a lot from one fabric to another, and plasma processes often benefit from being adapted specifically to each textile type. In spite of these variations, textiles have in common the fact that their surface is very often covered by a substantial layer of oils and sizes. These may be introduced during various steps in the production chain (i.e., fiber spinning, weaving, etc). Cleaning is therefore a very important step preceding the deposition of a thin film with plasma-enhanced chemical vapor deposition (PECVD) or sputtering, for instance. Usually, this can be adequately done by etching with O-containing plasmas. An example is shown in Fig. 1 for polyester yarns [3]. The yarns are cleaned in situ with $\mathrm{He} / \mathrm{O}_{2} \mathrm{RF}$ plasma (pressure of 7 mbar) prior to their metallization with Ag by magnetron sputtering. The quantity of residual oil at the surface of the untreated yarns is about $1.2 \mathrm{wt} \%$. As shown in Fig. 1, this can be effectively reduced by about two orders of magnitude with the plasma cleaning step. Not surprisingly, the quality of the Ag coating (conductivity, adhesion, and wash-fastness) scales with the yarn cleanliness. Figure 1 also shows that a wet-chemical industrial cleaning of the yarns can be used to decrease the plasma cleaning step duration. Nevertheless, the industrial washing does not remove all the oil, and plasma pre-cleaning leads to a marked improvement of the Ag coating. Beyond removal of oil and sizes at the surface of the yarns, plasma treatment also improves the adhesion of the Ag coating by functionalizing the polyester surface (grafting of O-containing polar groups [3,4]). The possibility of cleaning and functionalizing the yarn surface in situ before metallization is a clear advantage of plasma processes in comparison to other technologies (including wet-chemical electroless coating). With a suitable design of the plasma cleaning and sputtering system, an integrated air-to-air process has been developed, which allows Ag coating of polyester yarns at a speed of a few hundred meters per minute. The coated yarns have a relatively good conductivity (resistance $\sim 10-20 \Omega / \mathrm{cm}$ ) and are wash-fast. The metallization of yarns and fabrics currently receives much interest for various textile-based applications, ranging from antistatic garments to textile-integrated electronics [5-7].

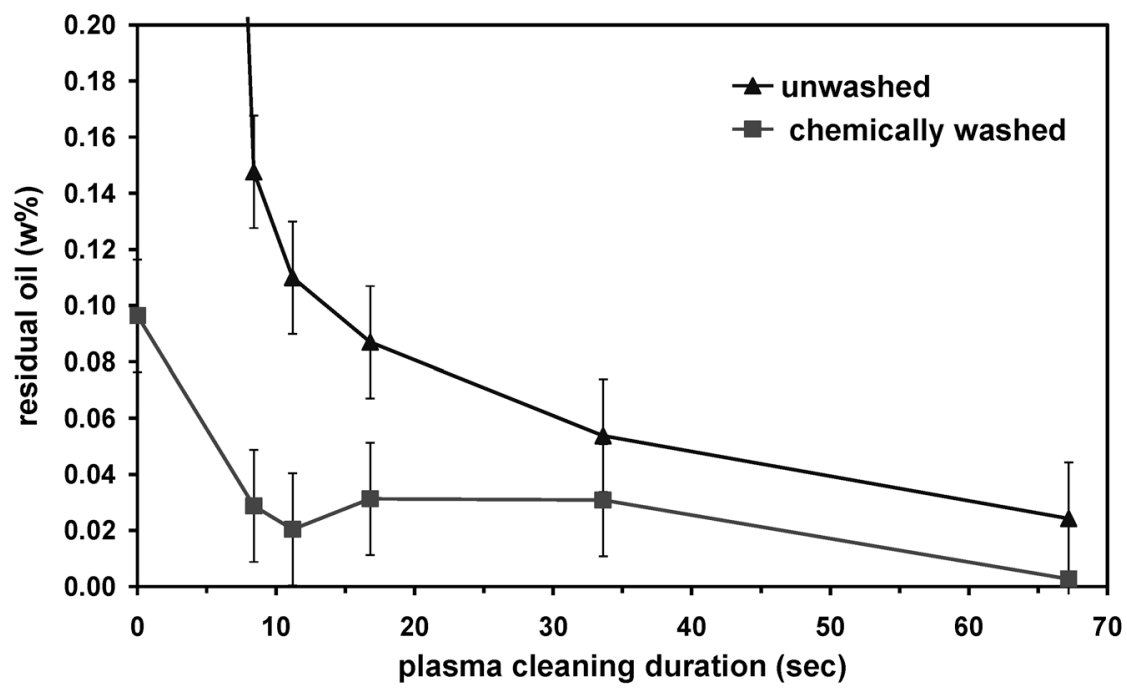

Fig. 1 Quantity of residual oil on polyester yarns as a function of the plasma cleaning duration. Unwashed yarns are compared to yarns that were washed in a detergent solution before the plasma cleaning [3]. 


\section{ENHANCEMENT OF WICKING PROPERTIES}

Wicking or liquid transport in textiles mainly depends on the capillary action in the interstices between individual fibers [8,9]. This is an important property for so-called moisture management garments, where the fabric is designed to achieve a rapid transport of sweat. It is possible to enhance the wicking properties of a textile with plasma treatments under low pressure. The latter are then used to increase surface hydrophilicity inside the textile capillary structure. For that purpose, the diffusion of the active plasma species in the interstices between the filaments is obviously very important. This effect is strongly influenced by the plasma parameters: the use of lower gas pressure increases the mean free path of the active species generated in the plasma and generally allows more efficient treatment of the inner fiber surfaces [10]. On the other hand, the density of active plasma species increases with rising pressure. This generally leads to faster surface cleaning and activation, which is economically advantageous from an industrial point of view. The interplay between these two effects can lead to a necessary compromise, where an "optimal" pressure is determined at which both the penetration and the speed of the plasma treatment are reasonable for a given application. This is illustrated in Figs. 2 and 3 for the $\mathrm{Ar} / \mathrm{O}_{2}$ (gas ratio 4/1) radio-frequency (RF) plasma hydrophilization of a well-defined and clean polyester multifilament weave. A scanning electron micrograph (SEM) of the weave is shown in Fig. 4a.

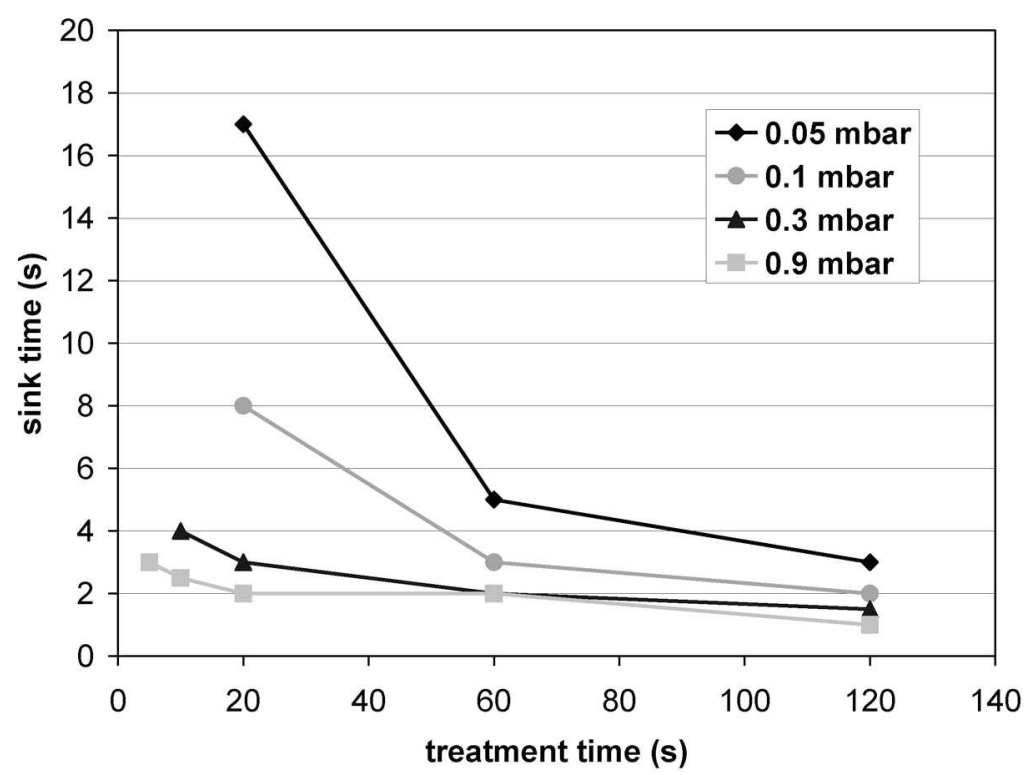

Fig. 2 Water droplet $(10 \mu \mathrm{l})$ sink time value as a function of the $\mathrm{Ar} / \mathrm{O}_{2}$ plasma treatment time and pressure for a polyester multifilament weave. 


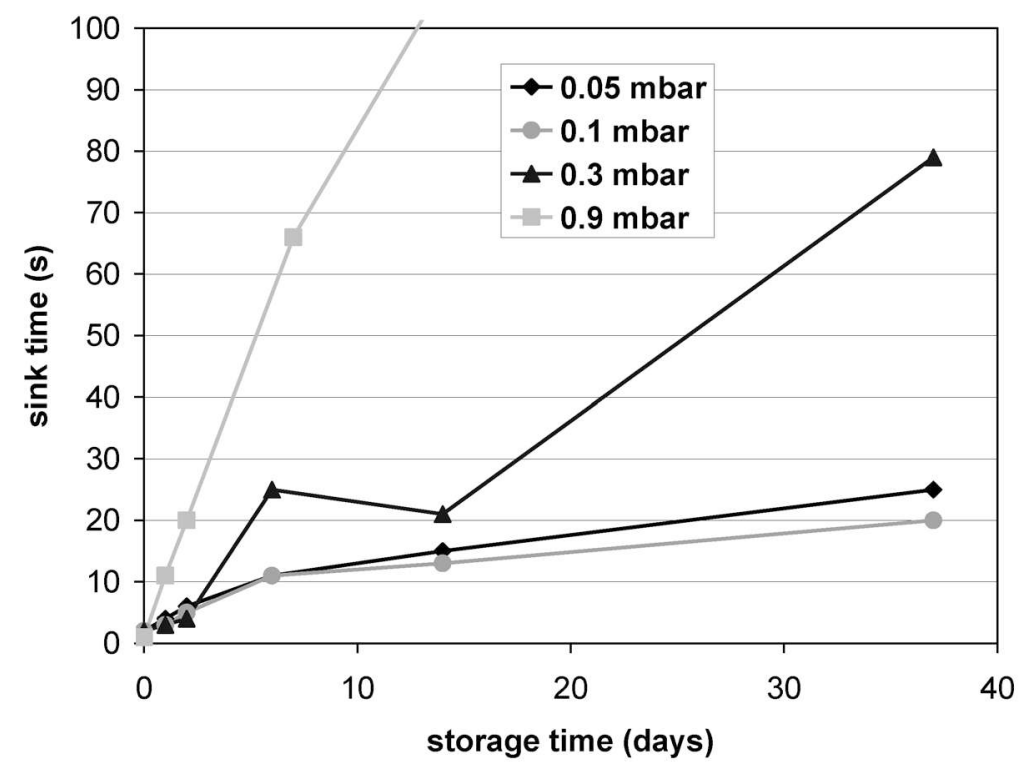

Fig. 3 Evolution of the water droplet sink time values with storage time in air $\left(65 \% \mathrm{RH}, 21{ }^{\circ} \mathrm{C}\right)$ after the $\mathrm{Ar} / \mathrm{O}_{2}$ plasma treatment of a polyester weave at different pressures. In all cases, a treatment duration of $300 \mathrm{~s}$ and a power of $400 \mathrm{~W}$ were used.

(a)

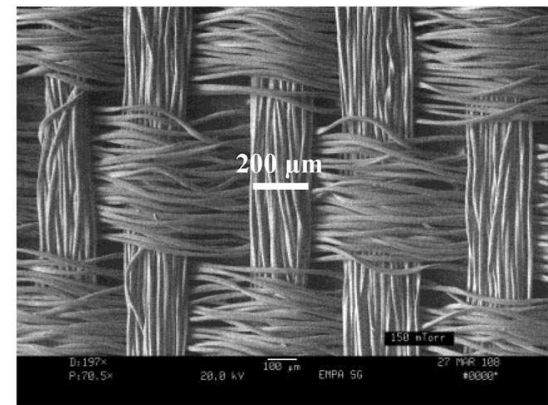

(b)

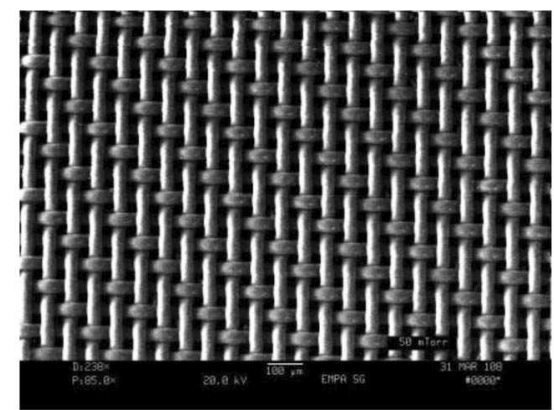

Fig. 4 SEM images of polyester (a) multifilament and (b) monofilament woven fabrics.

During these treatments, the samples were attached on the RF-driven (13.56 MHz) electrode of a symmetrical capacitive plasma reactor (web coater, described in details elsewhere [11]). The wicking properties of the treated fabrics were evaluated by measuring the sink time of a $10 \mu \mathrm{l}$ water droplet deposited on their surface. The sink time, which is defined as the time necessary for the complete ab- 
sorption of a droplet by the fabric, is about $600 \mathrm{~s}$ for untreated samples. Figure 2 shows that the plasma treatment time required to reduce the sink time to a given value decreases considerably upon increasing the pressure from 0.05 to $0.9 \mathrm{mbar}$ (for a constant plasma power of $400 \mathrm{~W}$ ). However, this pressure increase is also accompanied by a decrease of the gas particles mean free path (from $1 \mathrm{~mm}$ to about $50 \mu \mathrm{m}$ [10], i.e., significantly less than the overall diameter of the yarns as shown in Fig. 4a). This reduces the penetration of active species in the textile structure, the latter having a higher probability of losing energy due to an increased gas collision frequency. As pressure increases, the plasma treatment effect thus becomes more and more limited to the outer textile surface. As judged from Fig. 3, this apparently results in a lower stability of the improved wicking property imparted by the treatment. The increase of sink time values with storage time is attributed to the surface hydrophobic recovery of the plasma-modified polyester fibers. In the case of the samples treated at higher pressure (0.3-0.9 mbar), the hydrophilization of filaments that are not directly exposed at the surface of the weave is probably much weaker. This leads to a quick reduction of the capillary action after hydrophobic recovery, since only the topmost fibers retain a sufficient degree of hydrophilicity. Comparing Figs. 2 and 3, one could identify the pressure range between 0.1 and 0.3 mbar as being a good compromise between processing speed and treatment stability. Obviously, the definition of the optimal conditions will depend on the precise application. Likewise, the penetration of active plasma species in a textile will also strongly depend on its structure (thickness, compactness). These considerations are also relevant for other fields of application, including the functionalization of nonwoven textiles used in filtration devices.

One should note that other effects might also contribute to the differences observed here as a function of treatment pressure. For instance, the relative contributions of ion bombardment, VUV radiation and chemical etching are expected to vary with the pressure. Nevertheless, aging was found to be relatively independent of the treatment pressure in the case of polyester monofilament weaves. In this textile structure, most of the surfaces are directly exposed to the plasma (see Fig. 4b). This observation supports the assumption that the diffusion of plasma active species inside of the textile structure plays a decisive role in the case of the multifilament woven fabric.

\section{SURFACE FUNCTIONALIZATION WITH PLASMA POLYMERIZATION: NEW PERSPECTIVES}

Plasma polymerization offers an attractive possibility to deposit functional thin films on textiles [2]. By using appropriate combinations of gas mixtures and plasma parameters, a rather broad range of surface properties can be imparted to fabrics. In comparison to plasma activation (where no film is deposited), the surface properties resulting from plasma polymerization are relatively independent of the chemical nature of the textile substrate (although the latter has to be taken into account for the adhesion of the film $[12,13])$. Moreover, a high degree of cross-linking can give plasma polymer thin films an extra stability and limit adverse phenomena like hydrophobic recovery. The thickness of these films can be well controlled and kept within a range $(\sim 100 \mathrm{~nm})$ where the bulk properties of the textiles are not significantly altered.

For industrial applications, the use of relatively simple gas mixtures consisting of short-chain hydrocarbon monomer $\left(\mathrm{CH}_{4}, \mathrm{C}_{2} \mathrm{H}_{2}\right.$, or $\mathrm{C}_{2} \mathrm{H}_{4}$, for instance) and $\mathrm{N}$ - or O-containing reactive gases $\left(\mathrm{N}_{2}\right.$, $\mathrm{NH}_{3}, \mathrm{CO}_{2}$ ) can have practical and economical advantages. These processes are rather robust, and they do not require a liquid monomer introduction system, for instance [14]. These gas mixtures are suitable to deposit hydrophilic thin films that are resistant to aging and to washing. The deposition of thin films from $\mathrm{NH}_{3} / \mathrm{C}_{2} \mathrm{H}_{4}$ discharges is a particularly interesting example. For high $\mathrm{NH}_{3} / \mathrm{C}_{2} \mathrm{H}_{4}$ gas ratio, the films contain a high nitrogen concentration and show very hydrophilic properties [15]. Generally, films deposited from $\mathrm{NH}_{3} / \mathrm{C}_{2} \mathrm{H}_{4}$ mixtures were also shown to contain a good fraction of amino groups [16,17]. This is interesting, since amino groups have chemical properties that can be useful for various applications. They are known to promote cell adhesion [17], and their chemical activity can be used for the covalent attachment of functional molecules (see [18] and references therein). Within relatively broad 


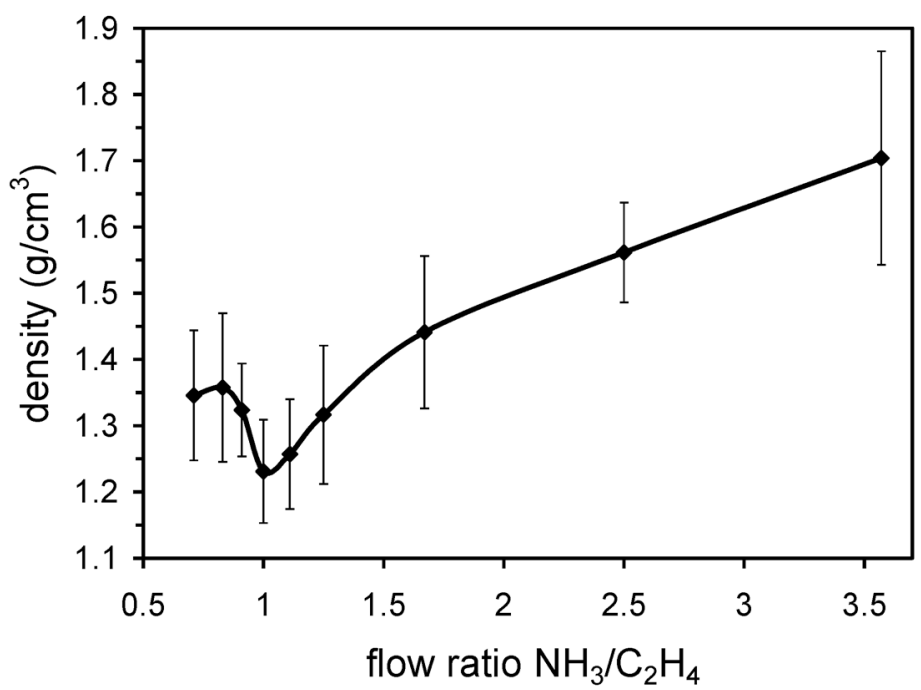

Fig. 5 Density of the $\mathrm{NH}_{3} / \mathrm{C}_{2} \mathrm{H}_{4}$ plasma polymer films as a function of the gas ratio.

$\mathrm{NH}_{3} / \mathrm{C}_{2} \mathrm{H}_{4}$ gas ratio and energy input (power/monomer flow) parameter ranges, a window has been identified where the resulting films have a lower density. As shown in Fig. 5, the film density generally rises with $\mathrm{NH}_{3} / \mathrm{C}_{2} \mathrm{H}_{4}$. This might be related to an increasing formation of $\mathrm{CN}$ radicals in the plasma, which reduces $\mathrm{H}$ passivation and increases the number of bonds between $\mathrm{C}$ and $\mathrm{N}$ in the film [19]. Nevertheless, a dip in the density vs. gas ratio curve is observed for $\mathrm{NH}_{3} / \mathrm{C}_{2} \mathrm{H}_{4}$ values around 1 . Although the reason for this is still unclear, it could be due to a preferential incorporation of $\mathrm{N}$ in the form of $-\mathrm{NH}_{x}$ network terminating groups [20]. These films can be regarded as nanoporous, and results have shown that their whole volume is available for the attachment of functional molecules [16]. These properties render possible a range of applications, where the coatings can be used as high specific surface area templates for the attachment of various functional molecules. Initial experiments on the anchoring of fluorocarbons have shown rather promising results. Coated textiles can be made oil-repellent, and the coatings are rather wash fast and abrasion-resistant.

The penetration of the film-forming plasma species in the textile structure is also often desired for applications based on plasma polymers. The above-mentioned trends as a function of the process pressure should therefore also be considered in the implementation of these processes. The deposition of plasma polymer films at a pressure of about 0.1 mbar usually leads to good results for textiles.

\section{CONCLUSION}

The implementation of plasma processes in the textile industry requires a careful consideration of the fabric properties (presence of oils, composition, and structure), and a choice of processing conditions that are adequate for industrial applications (simple gas mixtures, ease of operation, and optimized throughput for cost-efficiency). Besides environmental advantages, plasma technology also enables (sometimes unique) high-value-added textile products. Examples of processes that were successfully transferred to the industry have been outlined here: the production of conductive and wash-fast yarns, the permanent hydrophilization of fabrics and the functionalization of textiles with plasma polymerization. 


\section{REFERENCES}

1. R. Shishoo (Ed.). Plasma Technologies for Textiles, Woodhead Publishing Limited, Cambridge, UK (2007).

2. D. Hegemann. Indian J. Fibre Text. Res. 31, 99 (2006).

3. M. Amberg, C. Kasdallah, A. Ritter, D. Hegemann. J. Adhesion Sci. Technol. 24, 123 (2010).

4. L. J. Gerenser. J. Vac. Sci. Technol. A 8, 3682 (1990).

5. E. R. Post, M. Orth, P. R. Russo, N. Gershenfeld. IBM Syst. J. 39, 840 (2000).

6. D. Hegemann, M. Amberg, A. Ritter, M. Heuberger. Mater. Technol. 24, 41 (2009).

7. Q. Wei (Ed.). Surface Modification of Textiles, Woodhead Publishing Limited, Cambridge, UK (2009).

8. A. B. Nyoni, D. Brook. J. Text. Inst. 92, 119 (2006).

9. N. R. S. Hollies, M. M. Kaessinger, B. S. Watson, H. Bogaty. Text. Res. J. 27, 8 (1957).

10. H. U. Poll, U. Schladitz, S. Schreiter. Surf. Coat. Technol. 142-144, 489 (2001).

11. D. Hegemann. In Plasma Polymers and Related Materials, M. Mutlu (Ed.), University of Ankara (2005).

12. E. M. Liston, L. Martinu, M. R. Wertheimer. J. Adhesion Sci. Technol. 7, 1091 (1993).

13. D. Hegemann, H. Brunner, C. Oehr. Nucl. Instr. Methods Phys. Res., Sect. B 208, 281 (2003).

14. D. Hegemann, E. Körner, S. Guimond. Plasma Process. Polym. 6, 246 (2009).

15. M. M. Hossain, D. Hegemann, G. Fortunato, A. S. Herrmann, M. Heuberger. Plasma Process. Polym. 4, 471 (2007).

16. M. M. Hossain, A. S. Herrmann, D. Hegemann. Plasma Process. Polym. 4, S1068 (2007).

17. F. Truica-Marasescu, M. R. Wertheimer. Plasma Process. Polym. 5, 44 (2008).

18. K. S. Siow, L. Britcher, S. Kumar, H. J. Griesser. Plasma Process. Polym. 3, 392 (2006).

19. H. Seo, J. H. Kim, K. H. Chung, J. Y. Kim, S. H. Kim, H. Jeon. J. Appl. Phys. 98, 043308 (2005).

20. J. Ni, W. Wu, X. Ju, X. Yiang, Z. Chen, Y. Tang. Thin Solid Films 516, 7422 (2008). 